\title{
Editorial
}

\section{Additional Value of PET/CT to Other Radionuclide Imaging in Diagnosis in Neuroblastoma}

\author{
Amr, $\mathbf{M}^{1}$ and Moustafa, $\mathbf{H}^{2}$. \\ ${ }^{l}$ Nuclear Medicine Unit, NCI, ${ }^{2}$ Nuclear Medicine Unit NEMROCK Center, \\ Cairo University. Egypt.
}

Nuclear Medicine imaging is needed in Neuroblastoma for proper staging of primary tumor identification and metastatic surveillance, and monitoring of patients response. Metaiodobenzylguanidine (MIBG) is an analog of catecholamine precursors which localizes to neuroblastoma in primary sites and in bone, bone marrow, and lymph nodes with high specificity (95-100\%). It helps to study of tumor uptake and residence time in order to decide and plan a treatment with high activities of radiolabelled $\mathrm{MIBG}^{(1)}$. Also in evaluation of tumor response to therapy by measuring the intensity of MIBG uptake and the number of focal MIBG uptake sites. Also the extent of MIBG uptake might have prognostic significance. In some cases MIBG scintigraphy yields the only evidence of residual disease in a small percentage of patients ${ }^{(33)}$. (Fig.1) Persistent MIBG positivity during and after induction therapy forebodes a poor outcome. However one of the drawbacks of MIBG imaging is that a considerable minority of tumors are not MIBG avid ${ }^{(2)}$, the use of MIBG scintigraphy in routine patient assessment is controversial; as a recent study showed that MIBG results did not alter staging or treatment for any of the patients studied. Also, recurrent NB may fail to show uptake in some patients with recurrence ${ }^{(3)}$.Common falsely positive findings: Increased diffuse physiological uptake in hyperplastic adrenal gland after contralateral adrenalectomy increased focal physiological uptakes in the urinary tract or bowel, Urine contamination or any other external contamination (salivary secretion $)^{(4,5)}$. Falsely negative MIBG findings: Lack of tumor-cell MIBG avidity in view of differentiation, necrosis or interfering drugs. Nonvisualization of lesions because of intense radiotracer uptake in normal liver, myocardium, salivary glands, intestines $^{(5,6)}$. 


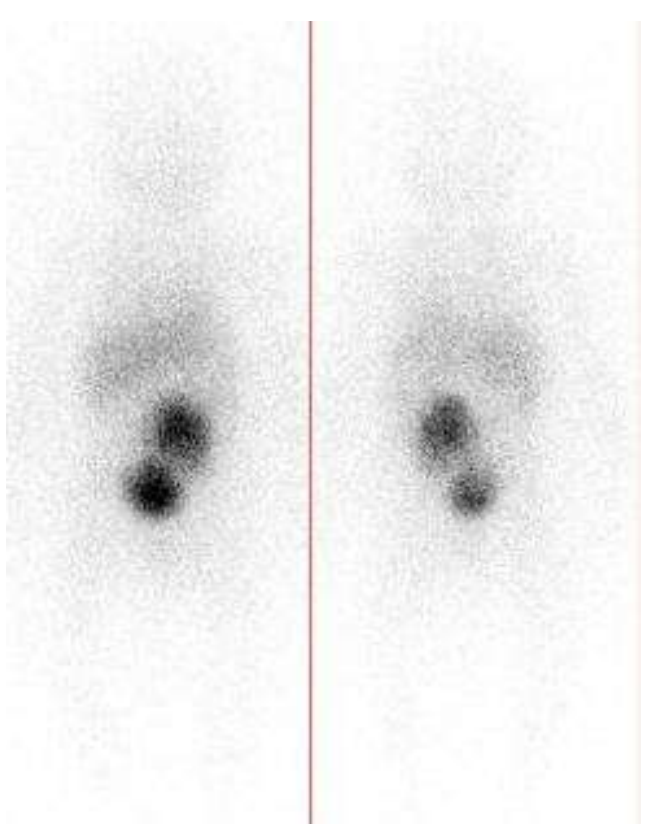

Fig (1): Iodinated MIBG in stage II NBL
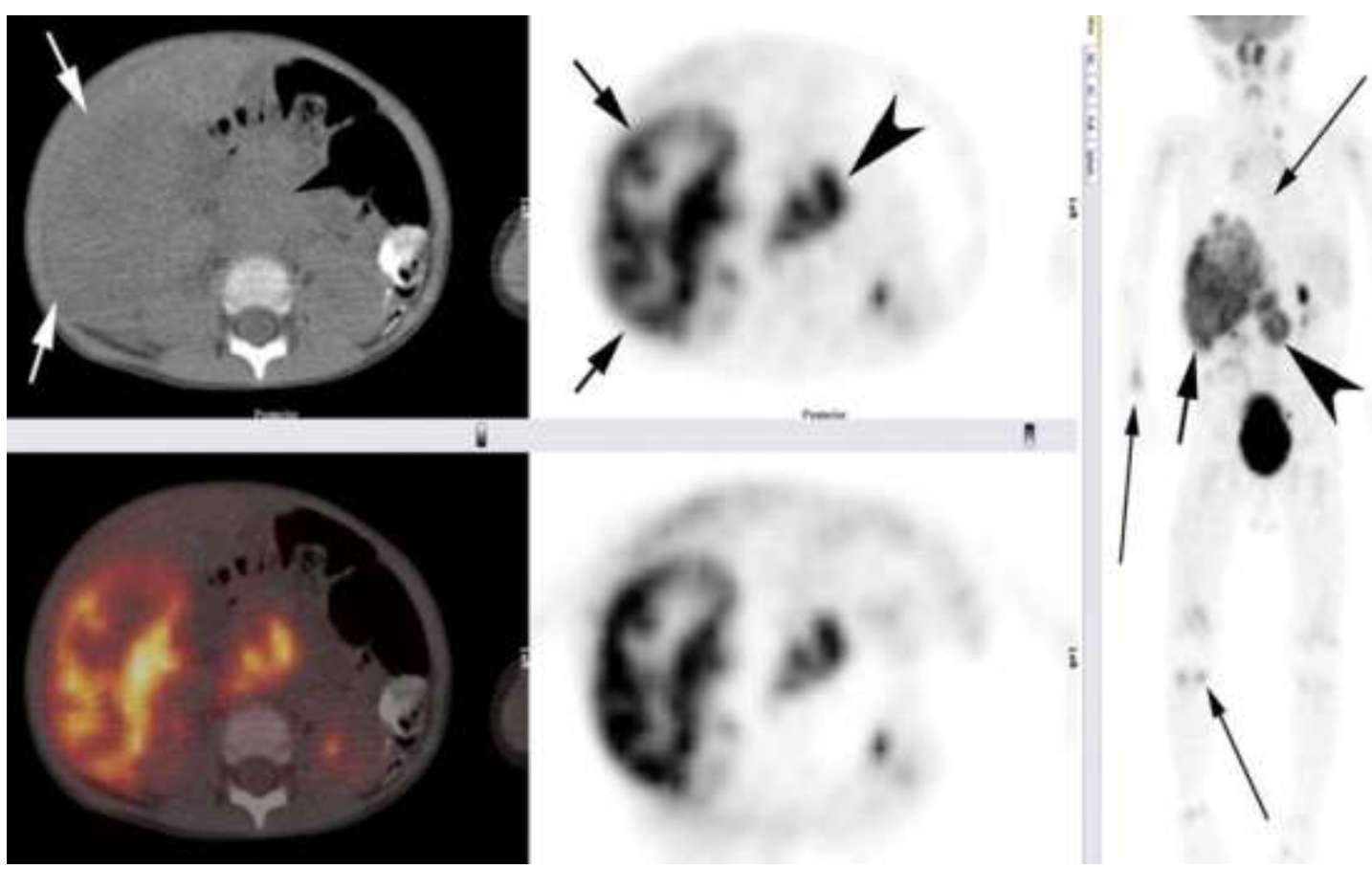

Fig. (2): Illustrates FDG tracer accumulation with the primary right suprarenal neuroblastoma associated with FDG avid loco-regional LNs. 
99mTc-MDP scans still have a role in evaluating newly diagnosed neuroblastoma patients since other scintigraphic modalities cannot distinguish between cortical bone and bone marrow involvement ${ }^{(7)}$. 99mTcMDP scans can be problematic because of a failure to detect lesions at the ends of longbones due to the intense uptake of bone-seeking agents in the normal growth plates of children. False-positive result is common with trauma. Many centers combine MIBG \& MDP in the diagnostic work up of children with NB to minimize the incidence of false positive and false negative results ${ }^{(8)}$. PET/CT in Neuroblastoma: PET exploits the increased aerobic glycolysis of malignant as compared with most normal cells, plus the retention within cells of the phosphorylated form of FDG. FDG uptake is, therefore, directly proportional to tumor burden and to tumor-cell proliferation. The capacity to characterize tumors both anatomically and metabolically sets PET a part from standard imaging modalities ${ }^{(9)}$.The published experience on neuroblastoma and PET is limited, yet PET scan findings appeared to correlate well with disease status as determined by MIBG scans, CT (or MRI), bone marrow tests, urine catecholamine levels, and clinical history. Sequential PET scans accurately depict treatment effects and disease evolution $^{(10)}$.
Because of the higher spatial resolution of the PET scanner and the tomographic nature of PET images, PET may be better than routine 131 I-MIBG scintigraphy for identifying small lesions and for delineating the extent or localizing anatomic sites of disease. (Fig. 2) PET might hold an advantage over MIBG scans for detecting metastases in liver, where the normally intense accumulation of MIBG can obscure disease ${ }^{(11)}$. PET and MIBG scans show similar patterns of diffusely abnormal skeletal findings in patients with extensive bone marrow involvement, but neither imaging modality reliably detects minimal bone marrow disease, PET shows more osteomedullary abnormalities that matches or surpasses MIBG scans ${ }^{(11,12)}$.PET may also yield useful clinical information in neuroblastoma patients beyond anatomic localization of disease. Through its depiction of the metabolic state of tumor cells, PET might provide insights into the proliferative or malignant potential of disease. Whether the degree of uptake at diagnosis has prognostic significance, especially with localized tumors, has not been studied. The findings in patients with metastatic neuroblastoma can influence treatment decisions. For example, in patients receiving cytotoxic therapy, but with persistence of measurable lesions by standard staging studies, PET scans with normal or with faintly abnormal distribution of FDG might be indicative of quiescent or responding, rather than. 
Actively proliferating or aggressive, disease; the impact would be support for continuation of the treatment program. FDG-PET drawbacks: Lack of visualization of lesions in the cranium because of high physiologic activity in brain, Increased FDG uptake in gut, thymus, urinary tract, sites of inflammation (skin, lungs, liver, recent sites of surgery), and hyperactive bone marrow are well-recognized nonmalignant causes or sites of FDG accumulation Falsely positive FDG uptake in the neck and shoulder region has been attributed to muscle activity but may also occur from accumulation in brown adipose tissue ${ }^{(13)}$. Clinical history $\&$ pattern of PET/CT lesions help to prevent mis-interpretation of falsely

\section{REFRENCES:}

1. Okazaki T, Kohno S, Mimaya J etal .Neuroblastoma detected by mass screening: the Tumor Board's role in its treatment. Pediatr SurgInt 2004; 20: 27-32.

2. Timmers HJ, Chen CC, Carrasquillo JA et al. Comparison of ${ }^{18} \mathrm{~F}$-fluoro-LDOPA, ${ }^{18} \mathrm{~F}$ fluoro-deoxyglucose, and ${ }^{18}$ F-flurodopamine PET and ${ }^{123} \mathrm{I}-$ mIBG scintigraphy in the localization of phaeochromocytoma and paraganglioma. J. Clin. Endocrinol. Metab.2009; 94, 4757-4767.

3. Andrich MP, Shalaby-Rana E, Movassaghi N, et al. The role of 131 iodine-metaiodobenzylguanidine scanning in the correlative imaging of patients with positive findings of PET. For example, in neuroblastoma patients, there should be little difficulty in distinguishing between inflammatory versus malignant causes of lung lesions seen by PET and CT: These patients often have documented or suspected systemic infections consequent to myelo-suppressive therapy, and neuroblastoma rarely metastasizes to lungs, especially when disease is responding to treatment. There should also be little difficulty in recognizing a benign cause for the diffuse FDG uptake in skeletal structures that can occur with cytokine-induced enhanced hematopoiesis (hyperactive bone marrow) after chemotherapy (and no morphologic evidence of neuroblastoma in bone marrow specimens) ${ }^{(13,14)}$.

neuroblastoma Pediatrics 1996; 97: 246-250.

4. Okuyama C, Sakane N, Yoshida T, et al. (123) I- or (125) Imetaiodobenzylguanidine visualization of brown adipose tissue. J Nucl Med.2002 Sept; 43 (9) 123440.

5. Peggi L, Liberti E, Pansini G, et al. Pitfalls in Scintigraphic detection of neuroendocrine tumors. Eur J Nucl Med 1992; 19:214-218.

6. Biasotti S, Garavanta A, Villavecchia GP, et al. False-negative metaiodobenzylguanidinescintigrapy at diagnosis of neuroblastoma. Med Pediatr Oncol. 2000; 35:153-155. 
7. Gordon I, Peters AM, Gutman A, etal. Skeletal assessment in neuroblastoma: the pitfalls of iodine123-MIBG scans. J Nucl Med. 1990; 31:129-134.

8. Turba E, Fagioli G, Mancini AF, et al. Evaluation of stage 4 neuroblastoma patients by means of MIBG and 99mTc-MDP scintigraphy. JNuclBiol Med. 1993; 37:107-114.

9. Schoder H, Erdi YE, Larson SM, Yeung HWD. PET/CT: a new technology innuclear medicine. Eur J Nucl Med Mol Imaging. 2003; 30:1419-1437.

10. Kushner BH. Neuroblastoma: a disease requiring a multitude of imaging studies. J Nucl Med 2004; 45: 1172-88.

11. Kushner BH, Yeung HWD, Larson $\boldsymbol{S M}$, et al. Extending PET scan utility to high-risk neuroblastoma: $18 \mathrm{~F}$ Fluorodeoxyglucose positron emission tomography as sole imaging modality in follow-up of patients. $J$ ClinOncol. 2001; 19:3397-3405.

12. Kushner BH, Yeh SDJ, Kramer K, et al. Impact of MIBG scintigraphy on assessing response of high-risk neuroblastoma to dose-intensive induction chemotherapy. $J$ Clin Oncol. 2003; 21: 1082-1086.

13. Yeung HWD, Grewal RK, Gonen M, et al. Patterns of 18F-FDG uptake in adipose tissue and muscle: a potential source of false positives for PET. $J$ Nucl Med. 2003; 44: 1789-1796.

14. BrisseH, McCarville B, Granata C. Guidelines for Imaging and Staging of Neuroblastic Tumors: Consensus Report from the International Neuroblastoma Risk Group Project 1. Radiology. 2011; 261 (1). 